\title{
Adaptive Output Feedback Based on Closed-Loop Reference Models for Hypersonic Vehicles
}

\author{
Daniel P. Wiese* and Anuradha M. Annaswamy ${ }^{\dagger}$ \\ Massachusetts Institute of Technology, Cambridge, MA 02139, USA \\ Jonathan A. Muse ${ }^{\ddagger}$ and Michael A. Bolender $\S$ \\ U.S. Air Force Research Laboratory, 2210 Eighth St., Wright-Patterson Air Force Base, Ohio 45433, USA \\ Eugene Lavretsky $₫$ \\ The Boeing Company, Huntington Beach, CA 92647, USA
}

\begin{abstract}
This paper presents a new method of synthesizing an output feedback adaptive controller for a class of uncertain, non-square, multi-input multi-output systems that often occur in hypersonic vehicle models. The main challenge that needs to be addressed is the determination of a corresponding square and strictly positive real transfer function. This paper proposes a new procedure to synthesize two gain matrices that allows the realization of such a transfer function, thereby allowing a globally stable adaptive output feedback law to be generated.

The unique features of this output feedback adaptive controller are a baseline controller that uses a Luenberger observer, a closed-loop reference model, manipulations of a bilinear matrix inequality, and the KalmanYakubovich Lemma. Using these features, a simple design procedure is proposed for the adaptive controller, and the corresponding stability property is established. The proposed adaptive controller is compared to the classical multi-input multi-output adaptive controller.

A numerical example based on a 6 degree-of-freedom nonlinear, scramjet powered, blended wing-body generic hypersonic vehicle model is presented. The adaptive output feedback controller is applied to result in stable tracking of uncertainties that destabilize the baseline linear output feedback controller.
\end{abstract}

\section{Introduction}

A state feedback linear quadratic regulator (LQR) baseline controller with integral action and augmented with an adaptive component has proven to be an effective choice for accommodating the parametric uncertainties present in flight control applications, and ensuring satisfactory reference tracking. ${ }^{1-7}$ However, such a controller requires that the state is measurable, which may not always be possible. Also, inaccuracies in the system output measurements may render state feedback controllers sensitive to measurement errors and thus not applicable. For these reasons there has been an increasing drive to develop an adaptive output feedback extension of the robust integral-augmented LQR baseline plus adaptive controller.

Existing classical methods of multi-input multi-output (MIMO) adaptive control are applicable for plants that are square. An $m \times m$ transfer matrix is used to represent the dynamic behavior of the plant, and the existence of a stable adaptive solution depends on the available prior information about this plant transfer matrix. ${ }^{8,9}$ The solution relies on non-minimal controller representations to dynamically decouple the plant, and the controller structure consists of a feedforward gain and two filters in the feedback path, the order of which depends on $m$ and an upper bound on

\footnotetext{
* Graduate Student, Mechanical Engineering, 77 Massachusetts Avenue Rm 3-441, Student Member AIAA

† Senior Research Scientist, Mechanical Engineering, 77 Massachusetts Avenue Rm 3-348, Member AIAA

${ }^{\ddagger}$ Research Aerospace Engineer, Autonomous Control Branch, Senior Member AIAA

$\S$ Senior Aerospace Engineer, Autonomous Control Branch, Associate Fellow AIAA

ๆ Boeing Senior Technical Fellow, Associate Fellow AIAA
} 
the observability index of the plant, $\nu$. The resulting classical MIMO adaptive solution will introduce $2 m \nu$ controller states and $2 m^{2} \nu$ adjustable parameters.

More recent methods of MIMO output feedback adaptive control have adopted a Luenberger observer-based approach in which a minimal observer is used to generate a state estimate to use for feedback control. ${ }^{7,10-12}$ This observer also serves as the reference model which is used by the adaptive controller, and the presence of the observer feedback gain $L$ provides the structure known as the closed-loop reference model, or CRM. ${ }^{13-16}$ These CRM based approaches have relied on the so-called squaring-up procedure ${ }^{17}$ to add fictitious inputs to a tall system (one where the dimension of the output is greater than the dimension of the input) making it square and ensuring any transmission zeros are stable. These fictitious inputs are used only to synthesize a postcompensator $S_{1}$ and the CRM gain $L$ which ultimately render a set of underlying error dynamics strictly positive real (SPR). These SPR error dynamics allowed stable update laws to be chosen to guarantee system stability. We note that systems with transmission zeros cannot be squared up using the method as described in Reference [17], which has led to a recent modification to overcome this limitation and allow the design of output feedback controllers for systems with stable transmission zeros. ${ }^{18}$

The CRM based output feedback design procedure proposed in this paper takes an alternative approach to synthesizing $S_{1}$ and $L$ which does not require the system first be squared-up. Instead, the postcompensator $S_{1}$ is determined as a generalized inverse of the system matrices, and a state feedback approach is used to stabilize a related lower order plant subsystem. This results in a feasible linear matrix inequality (LMI) which is solved to yield $L$. We consider in this work the case of tall systems, but the case of wide systems holds by duality. Furthermore, because $L$ is a component of both the baseline and adaptive controllers, it is crucial that it be selected to provide good frequency domain properties for the baseline control system, as well as desirable adaptive control performance. This procedure is able to exploit the structure of the given system to obtain a large amount of freedom in the selection of $L$ in order to achieve a robust baseline control design and the desired adaptive performance.

In Section II we introduce the class of uncertain plants which we wish to control, propose a control architecture, and formulate the control problem of interest. In Section III we provide some preliminaries which will be used in the synthesis of a globally stable controller for the uncertain system. Section IV provides a constructive procedure for obtaining an update law for an adaptive controller which guarantees global stability, and compares the proposed controller to the existing classical controller. Finally, a numerical example is presented in Section V to illustrate the efficacy of this method when applied to a 6-DOF nonlinear generic hypersonic vehicle (GHV) model. ${ }^{6,19}$

\section{Control Problem Formulation}

Consider the following MIMO uncertain open-loop system

$$
\begin{aligned}
\dot{x}_{p} & =A_{p} x_{p}+B_{p}\left(\Lambda u+\Psi_{p}^{\top} x_{p}\right) \\
y_{p} & =C_{p} x_{p} \\
z & =C_{p z} x_{p}+D_{p z}\left(\Lambda u+\Psi_{p}^{\top} x_{p}\right)
\end{aligned}
$$

where $A_{p} \in \mathbb{R}^{n_{p} \times n_{p}}, B_{p} \in \mathbb{R}^{n_{p} \times m}, C_{p} \in \mathbb{R}^{\ell \times n_{p}}, C_{p z} \in \mathbb{R}^{n_{e} \times n_{p}}, D_{p z} \in \mathbb{R}^{n_{e} \times m}$ are constant known matrices. The nonsingular matrix $\Lambda \in \mathbb{R}^{m \times m}$ and $\Psi_{p} \in \mathbb{R}^{m \times n_{p}}$, which represents constant matched uncertainty weights that enter the system through the columns of $B_{p}$, are unknown. The measured output is $y_{p}$, the regulated output is $z$, and the number of regulated outputs cannot exceed the number of inputs, that is $n_{e} \leq m$. The goal is to design a control input $u$ which will make $z$ track the reference command $z_{\text {cmd }}$ with bounded errors in the presence of the uncertainties $\Lambda$ and $\Psi_{p}$. We make the following assumptions about the system in (1).

\section{Assumption 1}

A) $\left(A_{p}, B_{p}\right)$ is controllable.

B) $\left(A_{p}, C_{p}\right)$ is observable.

C) $B_{p}, C_{p}$, and $C_{p} B_{p}$ are full rank.

D) Any finite transmission zeros of $\left(A_{p}, B_{p}, C_{p}, 0\right)$ are strictly stable, and the rank of the following matrix is full

$$
\operatorname{rank}\left(\left[\begin{array}{cc}
A_{p} & B_{p} \\
-C_{p z} & D_{p z}
\end{array}\right]\right)=n_{p}+n_{e}
$$


E) (a) $\Lambda$ is nonsingular and diagonal with entries of known sign

(b) $\left\|\Psi_{p}\right\|_{2}<\Omega<\infty$, where $\Omega$ is known

In order to facilitate command tracking, we introduce integral action, and for this purpose an additional state $x_{e}$ is defined as

$$
\dot{x}_{e}=z_{\mathrm{cmd}}-z
$$

This integral error state is appended to the plant in (1) leading to the following augmented open-loop dynamics

$$
\begin{aligned}
& {\left[\begin{array}{l}
\dot{x}_{p} \\
\dot{x}_{e}
\end{array}\right]=\left[\begin{array}{cc}
A_{p} & 0 \\
-C_{p z} & 0
\end{array}\right]\left[\begin{array}{l}
x_{p} \\
x_{e}
\end{array}\right]+\left[\begin{array}{c}
B_{p} \\
-D_{p z}
\end{array}\right]\left(\Lambda u+\Psi_{p}^{\top} x_{p}\right)+\left[\begin{array}{l}
0 \\
I
\end{array}\right] z_{\mathrm{cmd}}} \\
& {\left[\begin{array}{l}
y_{p} \\
x_{e}
\end{array}\right]=\left[\begin{array}{cc}
C_{p} & 0 \\
0 & I
\end{array}\right]\left[\begin{array}{l}
x_{p} \\
x_{e}
\end{array}\right]}
\end{aligned}
$$

The system in (3) can be written more compactly as follows

$$
\begin{aligned}
& \dot{x}=A x+B\left(\Lambda u+\Psi^{\top} x\right)+B_{\mathrm{cmd}} z_{\mathrm{cmd}} \\
& y=C x
\end{aligned}
$$

where $\Sigma=(A, B, C, 0)$, and where $A \in \mathbb{R}^{n \times n}, B \in \mathbb{R}^{n \times m}, B_{\text {cmd }} \in \mathbb{R}^{n \times n_{e}}$, and $C \in \mathbb{R}^{p \times n}$ are the known matrices given by

$$
A=\left[\begin{array}{cc}
A_{p} & 0_{n_{p} \times n_{e}} \\
-C_{p z} & 0_{n_{e} \times n_{e}}
\end{array}\right] \quad B=\left[\begin{array}{c}
B_{p} \\
-D_{p z}
\end{array}\right] \quad B_{\mathrm{cmd}}=\left[\begin{array}{c}
0_{n_{p} \times m} \\
I_{n_{e} \times n_{e}}
\end{array}\right] \quad C=\left[\begin{array}{cc}
C_{p} & 0_{\ell \times n_{e}} \\
0_{n_{e} \times n_{p}} & I_{n_{e} \times n_{e}}
\end{array}\right]
$$

and where $\Psi=\left[\begin{array}{ll}\Psi_{p}^{\top} & 0_{m \times n_{e}}\end{array}\right]^{\top}$ is unknown. Note that $p=\ell+n_{e}$. We make the following assumptions about the system $\Sigma$ in (4), which are equivalent to Assumption 1 related to the plant in (1):

\section{Assumption 2}

a) $(A, B)$ is controllable.

b) $(A, C)$ is observable.

c) $B, C$, and $C B$ are full rank.

d) Any finite transmission zeros of $\Sigma$ are strictly stable.

e) (a) $\Lambda$ is nonsingular and diagonal with entries of known sign

(b) $\|\Psi\|_{2}<\Omega<\infty$, where $\Omega$ is known

f) $\Sigma$ is tall: $p>m$.

Remark 1 The system in (1) satisfying Assumption 1A-D when augmented with the integral error state as shown in (3) also satisfies Assumption 2a-d. In other words, under Assumption 1A-D, integral error augmentation does not destroy controllability, observability, or the rank conditions. Nor does it add any transmission zeros. ${ }^{10}$

Remark 2 Assumptions $2 \mathrm{a}$ and $2 \mathrm{~b}$ are standard. Assumption 2c implies that inputs and outputs are not redundant, as well as a MIMO equivalent of relative degree unity. Assumption $2 \mathrm{~d}$ is a standard requirement for output feedback adaptive control. Assumption $2 \mathrm{f}$ can be considered without loss of generality as the case of wide systems $p<m$ holds by duality. The case of square systems has been given in Reference [20] and is discussed in Section IV.

\section{A. Baseline Control Design}

The underlying problem here is to design a control input $u$ in (4) so that the closed-loop system has bounded solutions and $z$ tends to $z_{\text {cmd }}$ with bounded errors in the presence of the uncertainties $\Lambda$ and $\Psi$. In this section, we describe the baseline control design for the nominal case when there are no uncertainties present, that is when $\Lambda=I$ and $\Psi=0$. 
A controller along the lines of References [10-12] is proposed, as it leads to a low order robust controller. This controller includes a Luenberger observer together with LQR feedback control gains. As our ultimate goal is to develop an adaptive controller which in turn requires a reference model, we propose a control design where the reference model has components of an observer as well. In particular, we introduce a feedback component into the reference model, with the corresponding feedback gain $L$ chosen similar to a Luenberger gain, that is, so that it ensures adequate stability margins for the nominal closed-loop system. The resulting reference model is referred to as a closed-loop reference model (CRM) which has been shown recently to result in highly desirable transient properties ${ }^{13-16}$ and is described as follows:

$$
\begin{aligned}
\dot{x}_{m} & =A_{m} x_{m}+B_{\mathrm{cmd}} z_{\mathrm{cmd}}+L\left(y_{m}-y\right) \\
y_{m} & =C x_{m}
\end{aligned}
$$

where $A_{m}=A+B K_{x}^{\top}$ and $K_{x}$ is selected such that $A_{m}$ is Hurwitz. Furthermore, $K_{x}$ should be selected to provide the desired closed-loop performance of the nominal system. With such a $K_{x}$, we can propose the following baseline controller that can guarantee command tracking and a certain amount of stability margins for the nominal closed-loop system.

\section{B. Adaptive Controller}

$$
u_{\mathrm{bl}}=K_{x}^{\top} x_{m}
$$

With the baseline controller determined as above, the next step is to design an adaptive controller in the presence of $\Lambda \neq I$ and $\Psi \neq 0$. Suppose we augment the nominal controller in (6) as

$$
u=\left(K_{x}+\Theta(t)\right)^{\top} x_{m}
$$

where $\Theta(t)$ is to be determined by a suitable update law. The question is if the introduction of the parameter $\Theta$ as in (7) is sufficient to accommodate the parametric uncertainties. For this purpose, we introduce a matching condition as described in Remark 3 below.

Remark 3 (Matching condition) The selection of the reference model state matrix as $A_{m}=A+B K_{x}^{\top}$ guarantees the existence of a parameter $\Theta^{*}$ that satisfies the following matching condition.

$$
A_{m}=A+B \Psi^{\top}+B \Lambda\left(\Theta^{* \top}+K_{x}^{\top}\right)
$$

where $\Theta^{*}$ is given by

$$
\Theta^{* \top}=\left(\Lambda^{-1}-I\right) K_{x}^{\top}-\Lambda^{-1} \Psi^{\top}
$$

Given a system satisfying Assumption 2, the matching condition in Remark 3, and the proposed control architecture, the reference tracking control problem is reduced to selecting the CRM gain $L$ in (5) and a suitable adaptive law for updating $\Theta(t)$ in (7).

In summary, the problem that is addressed in this paper is the determination of an adaptive augmented robust baseline output feedback controller as in (7) to control the plant in (4) using the CRM/Observer as in (5). This in turn necessitates finding an adaptive law for adjusting $\Theta$ in (7) and the observer gain $L$ in (5). The main tools used for determining the adaptive controller involve the Kalman-Yakubovich ${ }^{9}$ and matrix elimination lemmas ${ }^{21}$ which help reduce the problem of finding $L$ to a state feedback problem of a related lower order subsystem. Preliminaries pertaining to these tools are described in Section III. The complete adaptive control design and the corresponding stability result are presented in Section IV, and a numerical example is presented in Section V.

\section{Preliminaries}

The following well-known lemma gives necessary and sufficient conditions to ensure that the system $(A, B, C, 0)$ is SPR.

Lemma 1 (Kalman-Yakubovic) Given the strictly proper transfer matrix $G(s)$ with stabilizable and detectable realization $(A, B, C, 0)$, where $A \in \mathbb{R}^{n \times n}$ is asymptotically stable, $B \in \mathbb{R}^{n \times m}$ and $C \in \mathbb{R}^{m \times n}$, then $G(s)$ is $S P R$ if and only if there exists a $P=P^{\top}>0$ such that

$$
\begin{array}{r}
A^{\top} P+P A<0 \\
P B=C^{\top}
\end{array}
$$


PROOF The proof can be found in Reference [22].

Corollary There exists a matrix $P=P^{\top}>0$ that satisfies (9) if and only if

$$
C B=(C B)^{\top}>0
$$

Furthermore, when (10) holds, all solutions of (9) are given by

$$
P=C^{\top}(C B)^{-\top} C+B^{\perp} X B^{\perp \top}
$$

where $X=X^{\top}>0$ is arbitrary and $B^{\perp} \in \mathbb{R}^{n \times(n-m)}$ is a basis for the orthogonal complement, or annihilator of B. That is $B^{\perp}$ must satisfy $B^{\top} B^{\perp}=0$.

PROOF The proof can be found in Reference [20].

Lemma 2 (Matrix Elimination) Given

$$
G+C^{\top} L^{\top} P+P L C<0
$$

where $G \in \mathbb{R}^{n \times n}, C \in \mathbb{R}^{p \times n}$, and $P=P^{\top} \in \mathbb{R}^{n \times n}$ is full rank, an $L \in \mathbb{R}^{n \times p}$ exists which satisfies (12) if and only if the following inequality holds

$$
C^{\top \perp \top} G C^{\top \perp}<0
$$

where $C^{\top \perp} \in \mathbb{R}^{n \times(n-p)}$ satisfies $C C^{\top \perp}=0$.

PROOF The proof can be found in Reference [21].

\section{Adaptive Control Design}

In this section we provide the process for selecting the CRM gain $L$ in (5) and the update law for $\Theta$ in (7). To accomplish the goal of reference tracking we take an approach which focuses on the error between the closed-loop plant and the reference model states, as opposed to each of these trajectories individually. Thus, the goal of reference tracking can be ensured by appropriately selecting the update law to drive this state error to zero. Similarly, we consider the error between the parameter $\Theta$ in (7) and $\Theta^{*}$ in Remark 3. The resulting state tracking error and parameter error, respectively, can be defined as

$$
\begin{aligned}
e_{x} & =x-x_{m} \\
\widetilde{\Theta} & =\Theta-\Theta^{*}
\end{aligned}
$$

The problem of finding an adaptive law for $\Theta$ that guarantees stability depends on the relationship between the two errors above. This relation, denoted as error model, in turn provides cues for determining the adaptive law. In the problem under consideration, the underlying error model can be described as

$$
\begin{aligned}
& \dot{e}_{x}=\left(A+L C+B \Psi^{\top}\right) e_{x}+B \Lambda \widetilde{\Theta}^{\top} x_{m} \\
& e_{y}=C e_{x}
\end{aligned}
$$

where $e_{y}$ is the measured output error. As mentioned earlier, the problem of finding a stabilizing adaptive controller is equivalent to finding an $L$ and an adaptive law for adjusting $\widetilde{\Theta}$ in (13). Determining a stable adaptive law for an error model as in (13) relies on properties of an underlying transfer function that is SPR, ${ }^{9}$ which in turn enables the use of Lemma 1 in Section III. However, the definition of SPR is restricted to square transfer functions. As such, for these properties to be applicable to the error model in (13), a suitable static postcompensator $S_{1} \in \mathbb{R}^{m \times p}$ has to be chosen such that

$$
S_{1} C\left(s I-A-L C-B \Psi^{\top}\right)^{-1} B \in \mathbb{R}_{p}^{m \times m}(s)
$$

where $\mathbb{R}_{p}(s)$ denotes the ring of proper rational transfer functions with coefficients in $\mathbb{R}$. That is the underlying transfer matrix is square, and therefore can be evaluated in terms of SPR properties. We therefore introduce a synthetic output error $e_{s}$ as

$$
e_{s}=S_{1} C e_{x}
$$


With this postcompensator, the underlying error model is modified as

$$
\begin{aligned}
\dot{e}_{x} & =\left(A+L C+B \Psi^{\top}\right) e_{x}+B \Lambda \widetilde{\Theta}^{\top} x_{m} \\
e_{s} & =S_{1} C e_{x}
\end{aligned}
$$

Thus, the design of an output feedback adaptive controller is reduced to selecting matrices $S_{1} \in \mathbb{R}^{m \times p}$ and $L \in \mathbb{R}^{n \times p}$ such that the error dynamics in (14) are SPR.

In Section IV-A we provide a procedure to construct $S_{1}$ and $L$. This procedure requires $S_{1}$ to be solved as a generalized inverse based on the matrices of $\Sigma$ in (4) alone. $L$ is found by satisfying Lemma 1 (Kalman-Yakubovich), the solution of which is reduced to a state-feedback problem of a lower-order plant subsystem which ultimately leads to a feasible LMI which is solved numerically to obtain $L$.

\section{A. Finding $S_{1}$ and $L$}

In this section we provide a method for selecting $S_{1}$ and $L$ which ensure the system in (14) is SPR. The conditions from Lemma 1 to ensure $\left(A+L C+B \Lambda \Psi^{\top}, B, S_{1} C\right)$ is SPR are given by

$$
\begin{aligned}
\left(A+L C+B \Psi^{\top}\right)^{\top} P+P\left(A+L C+B \Psi^{\top}\right) & <0 \\
P B & =\left(S_{1} C\right)^{\top}
\end{aligned}
$$

where, by the corollary to Lemma 1 , a $P$ exists which satisfies (16) if and only if $S_{1} C B=\left(S_{1} C B\right)^{\top}$.

\section{Finding $S_{1}$}

The matrix $S_{1}$ satisfying (16) can be computed as a generalized left inverse of $C B$ as

$$
S_{1}=\left((C B)^{\top} C B\right)^{-1}(C B)^{\top}
$$

Note that this choice of $S_{1}$ is not unique.

\section{Finding $L$}

The annihilator matrices $B^{\perp}$ and $C^{\top \perp}$ in Section III are not unique. In the following subsection we will use the notation $N$ and $M$ to represent particular annihilators that satisfy $N B=0, C M=0$, and a few additional desired properties. That is, $N$ represents a particular $B^{\perp \top}$ and $M$ a particular $C^{\top \perp}$. Given arbitrary annihilators $B^{\perp}$ and $C^{\top \perp}$ we provide a constructive process for obtaining $N$ and $M$, and use these matrices to find $L$. The inequality (15) is satisfied if the following inequality is satisfied

$$
(A+L C)^{\top} P+P(A+L C)+Q<0
$$

for

$$
\Psi B^{\top} P+P B \Psi^{\top}<Q
$$

Using (16), the inequality (19) can be written as

$$
\Psi S_{1} C+\left(\Psi S_{1} C\right)^{\top}<Q
$$

Note that $Q$ satisfying (20) is independent of $P$. Using Lemma 2, an $L$ exists satisfying (18) if and only if a $P$ exists which satisfies

$$
M^{\top}\left(A^{\top} P+P A\right) M<-M^{\top} Q M
$$

Using (11), $P$ is given by

$$
P=\left(S_{1} C\right)^{\top}\left(S_{1} C B\right)^{-\top} S_{1} C+N^{\top} X N
$$

Substituting (22) into (21) we obtain

$$
(N A M)^{\top} X N M+(N M)^{\top} X(N A M)<-M^{\top} Q M
$$


Thus, the problem of finding an SPR $L$ which satisfies (15) is now reduced to finding the matrix $X$ satisfying (23). An $X$ satisfying (23) specifies a $P$ as in (22) that reduces (15) to a feasible LMI in $L$. This feasible LMI can then be easily solved using any widely available numerical LMI solver.

Reference [20] gave the inequality (23) for a square system, suggesting that $X$ be obtained by solving this LMI numerically. However, it was shown in Reference [23] that for a square system, the eigenvalues of NAM are the transmission zeros of the system and the annihilators $N$ and $M$ can be always be selected such that $N M=I$. Given a square system with only stable transmission zeros, this selection reduces (23) to a Lyapunov equation where the matrix $N A M$ is stable, and the existence of $X>0$ satisfying this inequality is guaranteed. ${ }^{24}$ Thus, when the system $\Sigma$ in (4) is square, (23) can be solved to obtain $X$, and $P$ can be computed using (22). The inequality (15) can then be solved for $L$. For a non-square systems the matrix $N A M$ is not square, and so determining $X>0$ satisfying (23) requires additional steps.

DETERMINING A SIMILARITY TRANSFORM We will now define a similarity transform $\Xi$ that will allow annihilator matrices $N$ and $M$ in (23) to be computed given arbitrary annihilators $B^{\perp}$ and $C^{\top \perp}$. Defining $\Xi$ as ${ }^{25}$

$$
\Xi=\left[\begin{array}{lll}
B & F & C^{\top \perp}
\end{array}\right]
$$

it is always possible to choose $F \in \mathbb{R}^{n \times(p-m)}$ so that $\Xi$ is invertible and

$$
\begin{aligned}
C \Xi & =\left[\begin{array}{ll}
\bar{C} & 0_{p \times(n-p)}
\end{array}\right] \\
\Xi^{-1} B & =\left[\begin{array}{ll}
I_{m \times m} & 0_{m \times(n-m)}
\end{array}\right]^{\top}
\end{aligned}
$$

where $\bar{C} \in \mathbb{R}^{p \times p}$. Define

$$
\bar{A}=\Xi^{-1} A \Xi=\left[\begin{array}{c|c}
\bar{A}_{11} & \bar{A}_{12} \\
\hline \bar{A}_{21} & \bar{A}_{22} \\
\hline \bar{A}_{31} & \bar{A}_{32}
\end{array}\right]
$$

where $\bar{A}_{22} \in \mathbb{R}^{(p-m) \times(n-p)}$ and $\bar{A}_{32} \in \mathbb{R}^{(n-p) \times(n-p)}$. Define the following transformed eliminators $N_{0}$ and $M_{0}$ which satisfy $N_{0} \Xi^{-1} B=0_{(n-m) \times m}$ and $C \Xi M_{0}=0_{p \times(n-p)}$ as

$$
\begin{aligned}
N_{0} & =\left[\begin{array}{ll}
0_{(n-m) \times m} & I_{(n-m) \times(n-m)}
\end{array}\right] \\
M_{0} & =\left[\begin{array}{ll}
0_{(n-p) \times p} & I_{(n-p) \times(n-p)}
\end{array}\right]^{\top}
\end{aligned}
$$

Note that these choices are not unique. Define

$$
\begin{aligned}
& N=N_{0} \Xi^{-1} \\
& M=\Xi M_{0}
\end{aligned}
$$

Note that with the selection of $M_{0}$ in (27) and with $\Xi$ in (24) that $M=C^{\top \perp}$. The matrix $N M$ is given by

$$
N M=\left[\begin{array}{ll}
0_{(n-p) \times(p-m)} & I_{(n-p) \times(n-p)}
\end{array}\right]^{\top}
$$

The inverse of $\Xi$ is given by

$$
\Xi^{-1}=\left[\begin{array}{c}
R \\
N_{1} \\
N_{2}
\end{array}\right]
$$

where $R \in \mathbb{R}^{m \times n}, N_{1} \in \mathbb{R}^{(p-m) \times n}$ and $N_{2} \in \mathbb{R}^{(n-p) \times n}$, and $\Xi^{-1}$ must obviously satisfy

$$
\Xi^{-1} \Xi=\left[\begin{array}{c}
R \\
N_{1} \\
N_{2}
\end{array}\right]\left[\begin{array}{lll}
B & F & C^{\top \perp}
\end{array}\right]=I
$$


where the matrix $F$, and thus $\Xi^{-1}$ are yet to be determined. From this we have that $N_{2} B=0_{(n-p) \times m}, N_{2} F=$ $0_{(n-p) \times(p-m)}$, and $N_{2} C^{\top \perp}=I_{(n-p) \times(n-p)}$. With this choice of $\Xi$ the matrix $N A M$ can be expressed as

$$
N A M=\left[\begin{array}{c}
\bar{A}_{22} \\
\bar{A}_{32}
\end{array}\right]=\left[\begin{array}{ll}
0_{(n-m) \times m} & I_{(n-m) \times(n-m)}
\end{array}\right]\left[\begin{array}{c|c}
\bar{A}_{11} & \bar{A}_{12} \\
\hline \bar{A}_{21} & \bar{A}_{22} \\
\hline \bar{A}_{31} & \bar{A}_{32}
\end{array}\right]\left[\begin{array}{c}
0_{p \times(n-p)} \\
I_{(n-p) \times(n-p)}
\end{array}\right]
$$

Note that with the choice of $N M$ satisfying (30), we can partition $X$ as

$$
X=\left[\begin{array}{cc}
X_{11} & 0_{(p-m) \times(n-p)} \\
0_{(n-p) \times(p-m)} & X_{22}
\end{array}\right]
$$

where $X_{11} \in \mathbb{R}^{(p-m) \times(p-m)}$ and $X_{22} \in \mathbb{R}^{(n-p) \times(n-p)}$ and $X>0$ if $X_{11}>0$ and $X_{22}>0$. Evaluating $X N M$ we have

$$
X N M=\left[\begin{array}{c}
0_{(p-m) \times(n-p)} \\
X_{22}
\end{array}\right]
$$

And so (23) is equivalent to the following Lyapunov equation

$$
\bar{A}_{32}^{\top} X_{22}+X_{22} \bar{A}_{32}=-M^{\top} Q_{\epsilon} M
$$

for $Q_{\epsilon}>Q$. The matrix $F$ in (24) must be selected such that $\bar{A}_{32}$ is Hurwitz, thus allowing $X_{22}$ to be obtained as the solution to the Lyapunov equation in (34). $X_{11}>0$ can then be selected arbitrarily to specify $X$. Expanding (32) using (28) and (29) we have

$$
\left[\begin{array}{c}
\bar{A}_{22} \\
\bar{A}_{32}
\end{array}\right]=\left[\begin{array}{ll}
0_{(n-m) \times m} & I_{(n-m) \times(n-m)}
\end{array}\right]\left[\begin{array}{c}
R \\
N_{1} \\
N_{2}
\end{array}\right] A\left[\begin{array}{lll}
B & F & C^{\top \perp}
\end{array}\right]\left[\begin{array}{c}
0_{p \times(n-p)} \\
I_{(n-p) \times(n-p)}
\end{array}\right]
$$

From which we can see

$$
\bar{A}_{32}=N_{2} A C^{\top \perp}
$$

Recall that $N_{2}$ has to satisfy $N_{2} B=0$, and $N_{2} C^{\top \perp}=I$. To satisfy these two conditions we see that $N_{2}$ lies in the nullspace of $B^{\top}$ and so we have

$$
N_{2}=K B^{\perp \top}
$$

where $K \in \mathbb{R}^{(n-p) \times(n-m)}$. The last condition requires $K B^{\perp \top} C^{\top \perp}=I$. We can satisfy $N_{2} F=0$ by selecting $F$ to lie in the nullspace of $N_{2}$, where dimension of the nullspace of $N_{2}$ is equal to $p$. With this choice of $N_{2}$ we can express $\bar{A}_{32}$ as

$$
\bar{A}_{32}=K B^{\perp \top} A C^{\top \perp}
$$

The requirements described above are stated as: find $K \in \mathbb{R}^{(n-p) \times(n-m)}$ such that

$$
\begin{array}{rc}
K B^{\perp \top} C^{\top \perp}= & I_{(n-p) \times(n-p)} \\
\bar{A}_{32}=K B^{\perp \top} A C^{\top \perp} \quad \text { is Hurwitz }
\end{array}
$$

An Equivalent State Feedback Problem We continue by showing how the selection of $F$ which defines the similarity transform $\Xi$ in (24) depends on $N_{2}$ in (31), which depends on $K$ in (36) and (37), can be found by solving a state feedback problem. The requirement in (36) is that $K$ is a left inverse of the tall matrix $B^{\perp \top} C^{\top \perp}$. This matrix has full rank by Assumption 2c. The generalized inverse of a tall matrix $T \in \mathbb{R}^{(n-m) \times(n-p)}$ with full rank is given by

$$
T^{-}=T^{\dagger}+U\left(I_{(n-m) \times(n-m)}-T T^{\dagger}\right)
$$


where $U \in \mathbb{R}^{(n-p) \times(n-m)}$ is arbitrary. This gives a form of all $K$ satisfying (36) as

$$
K=\left(B^{\perp \top} C^{\top \perp}\right)^{\dagger}+U\left(I_{(n-m) \times(n-m)}-\left(B^{\perp \top} C^{\top \perp}\right)\left(B^{\perp \top} C^{\top \perp}\right)^{\dagger}\right)
$$

This can be simplified as

$$
\begin{aligned}
K & =\left(B^{\perp \top} C^{\top \perp}\right)^{\dagger}+U\left(I_{(n-m) \times(n-m)}-J\right) \\
J & =\left(B^{\perp \top} C^{\top \perp}\right)\left(B^{\perp \top} C^{\top \perp}\right)^{\dagger}
\end{aligned}
$$

where $J \in \mathbb{R}^{(n-m) \times(n-m)}$ is a rank $n-p$ matrix. Thus $\bar{A}_{32}$ is given by

$$
\bar{A}_{32}=\left[\left(B^{\perp \top} C^{\top \perp}\right)^{\dagger}+U\left(I_{(n-m) \times(n-m)}-J\right)\right] B^{\perp \top} A C^{\top \perp}
$$

which can be written

$$
\bar{A}_{32}=G+U H
$$

where $G \in \mathbb{R}^{(n-p) \times(n-p)}$ and $H \in \mathbb{R}^{(n-m) \times(n-p)}$ are given by

$$
\begin{aligned}
& G=\left(B^{\perp \top} C^{\top \perp}\right)^{\dagger} B^{\perp \top} A C^{\top \perp} \\
& H=\left(I_{(n-m) \times(n-m)}-J\right) B^{\perp \top} A C^{\top \perp}
\end{aligned}
$$

Selecting $U$ such that $\bar{A}_{32}$ is Hurwitz is possible in general if $\left(G^{\top}, H^{\top}\right)$ is controllable. The uncontrollable modes of $\left(G^{\top}, H^{\top}\right)$ correspond to the transmission zeros of $\Sigma .^{26}$ If the system has any unstable zeros, no $U$ can be found such that $\bar{A}_{32}$ is Hurwitz. If the system has stable transmission zeros, $\left(G^{\top}, H^{\top}\right)$ is stabilizable, and $U$ can be selected to stabilize the remaining modes. If the system has no transmission zeros, $\left(G^{\top}, H^{\top}\right)$ is controllable, and $U$ can be picked to make the poles of $\bar{A}_{32}$ arbitrarily. By Assumption $2 \mathrm{~d} \Sigma$ has no unstable transmission zeros, so $\left(G^{\top}, H^{\top}\right)$ will be at least stabilizable. With $U$ computed using the desired state-space technique, $\bar{A}_{32}$ is determined as in (40). $K$ can then be solved for from (38) and (39) and $N_{2}$ computed using (35). $F$ is then selected to lie in the nullspace of $N_{2}$, and to make $\Xi$ full rank. With this choice of $F$, the matrix $\Xi$ is completely specified, and $N$ can be solved for from (28) and $M$ given by $M=C^{\top \perp}$. Finally, (34) must be solved to obtain $X_{22}$, which requires the specification of $Q>0$. The following paragraph and theorem provide a method to select an appropriate $Q$.

Solving the LMI to ObTain $L$ All that remains to solve the LMI in (18) for $L$ is to specify $P$ as given by (22) and $Q$. Solving (34) for $X_{22}$ also requires $Q$, although this equation places no restriction on how $Q>0$ is selected. However, we must choose an appropriate $Q$ which guarantees the feasibility of the LMI in (18) by satisfying (20), as given by the following theorem.

Theorem 1 If $Q$ is chosen as

$$
Q=2 \Omega\left\|C_{s}\right\|_{2} I_{n \times n}
$$

where $C_{s}=S_{1} C$ and $\Omega$ is defined as in Assumption $1 E-(b)$, then (20) holds.

Proof Using $C_{s}=S_{1} C$ the inequality (20) can be written

$$
\Psi C_{s}+\left(\Psi C_{s}\right)^{\top}<Q
$$

Using $\Psi C_{s} \leq\left\|\Psi C_{s}\right\|_{2} I \leq\|\Psi\|_{2}\left\|C_{s}\right\|_{2} I<\Omega\left\|C_{s}\right\|_{2} I$ the matrix $Q$ in (43) satisfies (20).

With $Q$ picked as in (43) and $\bar{A}_{32}$ made stable by selection of $U$ in (40), the Lyapunov equation in (34) can be solved to obtain $X_{22}$. This procedure guarantees the feasibility of the LMI in (18) which can be solved numerically with any widely available solver. This procedure is summarized in the following subsection.

\section{B. Summary of the Design Procedure for $S_{1}$ and $L$}

Subsection IV-A provided a procedure to determine $S_{1}$ and $L$ for the system $\Sigma$ satisfying Assumption 2 which render (14) SPR. This subsection summarizes the overall procedure. Given known plant matrices $A, B, B_{\mathrm{cmd}}, C$, and uncertainties $\Lambda$ and $\Psi$ in (4), reference model in (5), and control law in (7), the following steps provide a procedure to determine $S_{1}$ and $L$ such that the underlying error dynamics in (14) are SPR: 
1. Solve for $S_{1}$ as in (17).

2. Determine arbitrary annihilators $B^{\perp}$ and $C^{\top \perp}$ such that $B^{\top} B^{\perp}=0$ and $C C^{\top \perp}=0$.

3. Calculate matrices $G$ and $H$ using (39), (41), and (42) and then solve for $U$ such that $\bar{A}_{32}$ in (40) is Hurwitz.

4. Compute $K$ using (38) and define $N_{2}$ as in (35).

5. Determine $F$ in $\operatorname{null}\left(N_{2}\right)$ such that $\Xi=\left[\begin{array}{lll}B & F & C^{\top \perp}\end{array}\right]$ is full rank.

6. Define $N_{0}$ as in (26). Calculate $N=N_{0} \Xi^{-1}$ and set $M=C^{\top \perp}$.

7. Select $Q$ as in (43) and solve (34) to obtain $X_{22}$. Assemble $X$ as shown in (33) where $X_{11}>0$ is arbitrary.

8. Solve for $P$ as in (22).

9. Solve the LMI in (18) to obtain $L$

Remark 4 In the case where $p-m \geq n-p$, we are ensured that $H$ in (40) is a matrix of full column rank and so $H^{\dagger} H=I_{(n-p) \times(n-p)}$. This provides the freedom in selecting $U$ to not only make $\bar{A}_{32}$ stable, but to select it to be any stable matrix. This allows us to select $X_{22}>0$ arbitrarily, and then solve for $\bar{A}_{32}^{*}$ as the solution to the Lyapunov equation $\bar{A}_{32}^{* \top} X_{22}+X_{22} \bar{A}_{32}^{*}=-M^{\top} Q_{\epsilon} M$. Then $U$ can be picked in step 3 as

$$
U=\left(\bar{A}_{32}^{*}-G\right) H^{\dagger}
$$

Remark 5 The calculation of $L$ should conclude with the verification that $A+L C+B K_{x}^{\top}$ is Hurwitz. While this is not a theoretical requirement, for practical implementation on systems such as the one presented in Section $\mathrm{V}$, this requirement is enforced to ensure the reference model in (5) is stable.

\section{Adaptive Law and Stability Proof}

Using the closed-loop reference model defined in (5) with $L$ selected as described in Section IV-A, we then propose the following update law:

$$
\dot{\widetilde{\Theta}}=-\Gamma x_{m}\left(S_{1} e_{y}\right)^{\top} \operatorname{sgn}(\Lambda)
$$

where $S_{1}$ is chosen using (17). Globally stability of the closed-loop system is guaranteed by the following theorem.

Theorem 2 Given the uncertain linear system in (4) which satisfies Assumption 2, the reference model in (5), and control law as in (7), the update law in (45) results in global stability, with $e_{x}(t) \rightarrow 0$ as $t \rightarrow \infty$.

PROOF Propose the following radially unbounded Lyapunov function candidate

$$
V\left(e_{x}, \widetilde{\Theta}\right)=e_{x}^{\top} P e_{x}+\operatorname{tr}\left(|\Lambda| \widetilde{\Theta}^{\top} \Gamma^{-1} \widetilde{\Theta}\right)
$$

using (45) the time derivative of $V$ along system trajectories can be evaluated as $\dot{V}=-e_{x}^{\top} \widehat{Q} e_{x} \leq 0$ where $\widehat{Q}>0$, and indeed the proposed candidate does serve as a Lyapunov function for this system. Furthermore, it can be shown using Barbalat's lemma that $e_{x}(t) \rightarrow 0$ as $t \rightarrow \infty$.

With $e_{x}(t) \rightarrow 0$ as $t \rightarrow \infty$, we achieve desired bounded reference tracking of $z_{\text {cmd }}$ by $z$. In the following section we demonstrate the efficacy of the proposed method by providing a numerical example, but first we examine the applicability of the CRM based method as compared to the classical MIMO adaptive control method.

\section{Comparison Between CRM based and Classical MIMO Adaptive Control}

Given the classical approaches used in the literature thus far, the obvious question that is raised is how the proposed MIMO controller fares compared to the classical ones. The first point to note here is that the classical approaches are limited to square plants while our approach proposed here is not. This is the most obvious advantage of our method. The next question that arises is a comparison of the two approaches when the underlying plant is square. This is addressed below.

As a first step, we provide relevant definitions below:

Definition 1 (Markov Parameters) [27] Given a transfer matrix G(s), the Markov Parameters are given by

$$
H_{0}=\lim _{s \rightarrow \infty} G(s), \quad H_{1}=\lim _{s \rightarrow \infty} s\left(G(s)-H_{0}\right), \quad H_{2}=\lim _{s \rightarrow \infty} s^{2}\left(G(s)-H_{0}-H_{1} s^{-1}\right)
$$

and so forth. 
Theorem 3 The set $(A, B, C, D)$ is a realization of $G(s)$ if and only if

$$
H_{0}=D \quad H_{i}=C A^{i-1} B, \quad i=1,2, \ldots
$$

Proof The proof can be found in Reference [27].

Definition 2 (Relative Degree One) The MIMO system $G(s)$ with realization $(A, B, C, D)$ is said to be Relative Degree One if $H_{0}=0$ and $H_{1}=C B$ is full rank.

Lemma 3 Reference [9] Given a square nonsingular strictly proper transfer matrix $W_{p}(s) \in \mathbb{R}_{p}^{m \times m}(s)$, its Hermite form is diagonal if and only if the constant matrix $E\left(W_{p}(s)\right)$ is nonsingular, where $E$ is calculated as follows. Calculate $r_{i}$ as the minimum relative degree in the $i^{\text {th }}$ row of $W_{p}(s)$ and the rows of $E$ are

$$
E_{i}=\lim _{s \rightarrow \infty} s^{r_{i}} W_{p, i}(s)
$$

where $W_{p, i}(s)$ corresponds to the $i^{\text {th }}$ row of $W_{p}(s)$.

PROOF The proof can be found in Reference [28]

Given $W_{p}(s) \in \mathbb{R}_{p}^{m \times m}(s)$, the assumptions that must be satisfied for a classical adaptive control solution to exist are as follows. ${ }^{9}$

\section{Assumption 3}

(i) The high frequency gain matrix $K_{p}$ is of the form $K_{p}=\bar{K}_{p} \Lambda$ where $\bar{K}_{p}$ is known and $\operatorname{sign}(\Lambda)$ is known.

(ii) The right Hermite normal form $H_{p}(s)$ of $W_{p}(s)$ is known.

(iii) An upper bound $\nu$ on the observability index of $W_{p}(s)$ is known.

(iv) The zeros of $W_{p}(s)$ lie in $\mathbb{C}^{-}$.

Theorem 4 Given the square plant $W_{p}(s) \in \mathbb{R}_{p}^{m \times m}$ with realization $(A, B, C, 0)$, the Hermite form $H_{p}(s)$ of $W_{p}(s)$ is diagonal if $C B$ is full rank. Furthermore, the high frequency gain matrix is given by $K_{p}=C B$.

PROOF Theorem 3 connects the Markov Parameters of Relative Degree One systems to the realization of $W_{p}(s)$ with $H_{0}=0$ and $H_{1}=C B$. With this and Definition 1 we have that $\lim _{s \rightarrow \infty} s W_{p}(s)=C B$ is full rank, and so the minimum relative degree in each row of $W_{p}(s)$ is $r_{i}=1$. By Lemma $3 E\left(W_{p}(s)\right)=C B$ and the Hermite form $H_{p}(s)$ of $W_{p}(s)$ is diagonal. In Reference [9] it is shown that $E\left(W_{p}(s)\right)=K_{p}$.

Using Definitions 1 and 2 as well as Theorems 3 and 4, we show in Proposition 1 that the classical and our CRM based MIMO adaptive control solution in this paper are equally applicable when the system in (1) is square.

Proposition 1 Consider the uncertain system in (1) where $\ell=m$ and the plant transfer matrix is given by

$$
W_{p}(s)=C_{p}\left(s I-A_{p}-B_{p} \Psi_{p}^{\top}\right)^{-1} B_{p} \Lambda
$$

if the plant in (1) satisfies Assumption 1, then the corresponding $W_{p}(s)$ in (47) satisfies Assumption 3.

Proof Assumption 1E-(a) and Theorem 4 can be shown to imply that the corresponding $K_{p}$ satisfies Assumption 3(i). Assumption $1 \mathrm{C}$ together with Theorem 4 implies that the corresponding Hermite form is diagonal with known entries and is therefore known, which leads to Assumption 3(ii). Assumption 3(iii) follows from the fact that $n_{p}$ is known. Finally Assumption 1D is equivalent to Assumption 3(iv).

In addition to the main advantage of our proposed method of applicability to non-square plants, our proposed controller is of lower order, requiring only $n$ controller states and $n m$ adjustable parameters, as compared with the classical solution which will introduce $2 m \nu$ states and $2 m^{2} \nu$ parameters. This reduces the number of states and parameters necessary by at least two, since $n \leq \nu m .{ }^{29}$ Finally our solution is based on a CRM, which has been shown to possess a superior transient performance. ${ }^{13-16}$

It should be noted that of Assumptions 1A-E, which are required to be satisfied for the proposed controller, the most restrictive one is Assumption 1C, which implies that the MIMO system must have Relative Degree One. In most aerial platforms including hypersonic vehicles, this assumption is easy to satisfy as the relative degree of the transfer functions between the control surface deflections and aircraft angular rates is unity. Additionally, the structure of the 
plant as in (1) which has matched uncertainties is also commonly present in flight control applications where much of the plant uncertainty is in the aerodynamic moment coefficients and loss of control effectiveness, which are spanned by the columns of $B$. It is however required that the uncertainty $\Psi_{p}$ satisfy Assumption 1E-(b), which is not required in the classical approach.

\section{Numerical Example}

We now present a numerical example using this output feedback design procedure applied to the control design for a 6-DOF GHV model. ${ }^{6,19}$ The GHV is a small blended wing-body vehicle, with 3-D inlet and nozzle, and axisymmetric through-flow scramjet engine. The nonlinear equations of motion describing the GHV are linearized about a nominal flight condition of Mach 6 at an altitude of 80,000 feet, corresponding to a dynamic pressure of 1474 psf. Modal analysis allowed the linearized equations of motion to be decoupled, and the resulting uncertain longitudinal and lateral-directional plant subsystems are represented as in Equation (1). In Reference [6] a state feedback LQR baseline controller with integral action and augmented with an adaptive component was applied to design two independent CRM based state feedback adaptive controllers - one for each of the plant subsystems. This approach was very effective at maintaining stability and tracking performance in the presence of uncertainty, but assumed availability of angle-of-attack and sideslip angle measurements. In this work, we no longer assume that these incidence angles are measurable, which is more realistic for this class of vehicle, thus turning the problem into one of output feedback. The adaptive control design procedure described in Section IV was used to design two independent CRM based output feedback adaptive controllers - one for each of the two plant subsystems.

\section{A. Longitudinal Subsystem}

The state, control, output, and regulated output for the linear longitudinal subsystem as represented in (1) are given by

$$
x_{p}=\left[\begin{array}{ll}
\alpha & q
\end{array}\right]^{\top} \quad u=\delta_{e} \quad y_{p}=q \quad z=q
$$

respectively, where $\alpha$ represents the angle of attack, and $q$ is the pitch rate. The control input $\delta_{e}$ represents the elevator deflection angle. The pitch rate is measurable but the angle of attack is not. The control goal is to track pitch rate commands $z_{\mathrm{cmd}}=q_{\mathrm{cmd}}$. The longitudinal subsystem satisfies Assumption 1. The state vector $x_{p}$ is augmented with the integral error state as in (2) resulting in a system of the form (4) which satisfies Assumption 2. The augmented state and output vector are

$$
x=\left[\begin{array}{lll}
\alpha & q & x_{e}
\end{array}\right]^{\top} \quad y=\left[\begin{array}{ll}
q & x_{e}
\end{array}\right]^{\top}
$$

The baseline control gain $K_{x}$ in (6) was computed resulting in the following state feedback gain and phase margin

$$
\begin{aligned}
& \mathrm{GM}_{\mathrm{sf}}=[-14.5,165.7] \quad \mathrm{dB} \\
& \mathrm{PM}_{\mathrm{sf}}=60 \mathrm{deg}
\end{aligned}
$$

The controller was then tuned by selecting $X_{11}>0$ and solving for $X_{22}$ in step 7 resulting in

$$
X=\left[\begin{array}{cc}
2 & 0 \\
0 & 13.3
\end{array}\right]
$$

This provided the following gain and phase margin for the resulting output feedback compensator

$$
\begin{aligned}
\mathrm{GM}_{\text {of }} & =[-14.2,33.2] \quad \mathrm{dB} \\
\mathrm{PM}_{\text {of }} & =59 \quad \mathrm{deg}
\end{aligned}
$$

The numerical values for the linear system matrices and LQR weighting matrices can be found in the appendix. 


\section{B. Lateral Subsystem}

The state, control, output, and regulated output for the linear lateral-directional subsystem as represented in (1) are given by

$$
x_{p}=\left[\begin{array}{llll}
\beta & p & r & \phi
\end{array}\right]^{\top} \quad u=\left[\begin{array}{ll}
\delta_{a} & \delta_{r}
\end{array}\right]^{\top} \quad y_{p}=\left[\begin{array}{lll}
p & r & \phi
\end{array}\right]^{\top} \quad z=\phi
$$

respectively, where $\beta$ represents the sideslip angle, $p$ the roll rate, $r$ the yaw rate, and $\phi$ the roll angle. The control inputs $\delta_{a}$ and $\delta_{r}$ represent the aileron and rudder deflection angles, respectively. All states are measurable except the sideslip angle. The control goal is to track roll angle commands $z_{\mathrm{cmd}}=\phi_{\mathrm{cmd}}$. The lateral-directional subsystem can be shown to satisfy Assumption 1. The state vector $x_{p}$ is augmented with the integral error state as in (2) resulting in a system of the form (4) which satisfies Assumption 2. The augmented state and output vector are

$$
x=\left[\begin{array}{lllll}
\beta & p & r & \phi & x_{e}
\end{array}\right]^{\top} \quad y=\left[\begin{array}{llll}
p & r & \phi & x_{e}
\end{array}\right]^{\top}
$$

The baseline control gain $K_{x}$ in (6) was computed resulting in the following state feedback gain and phase margin

$$
\begin{aligned}
\mathrm{GM}_{\mathrm{sf}} & =[-6.5,161] \mathrm{dB} \\
\mathrm{PM}_{\mathrm{sf}} & =60 \mathrm{deg}
\end{aligned}
$$

Since the conditions of Remark 4 are satisfied, $X_{22}$ can be selected arbitrarily. The controller was then tuned by selecting $X_{11}>0$ and $X_{22}>0$ resulting in

$$
X=\left[\begin{array}{lll}
2 & 0 & 0 \\
0 & 2 & 0 \\
0 & 0 & 2
\end{array}\right]
$$

This provided the following gain and phase margin for the resulting output feedback compensator

$$
\begin{aligned}
& \mathrm{GM}_{\mathrm{of}}=[-7.3,14.5] \mathrm{dB} \\
& \mathrm{PM}_{\text {of }}=48 \quad \mathrm{deg}
\end{aligned}
$$

The numerical values for the linear system matrices and LQR weighting matrices can be found in the appendix.

\section{Simulations}

The linear, adaptive augmented baseline controllers designed for the longitudinal and lateral-directional systems as described by (1) with reference model in (5), control law as in (7), and update law in (45) were then implemented in a simulation of the GHV model. Second order actuator dynamics were included in the simulation on the elevators, ailerons, and rudders. The numerical property values are listed in Table 1.

Table 1. Second order aerodynamic control surface actuator parameters

\begin{tabular}{llc}
\hline Parameter & Unit & Value \\
\hline Surface deflection limit & {$[\mathrm{deg}]$} & -30 to 30 \\
Surface rate limit & {$[\mathrm{deg} / \mathrm{s}]$} & -100 to 100 \\
Damping ratio $\zeta$ & & 0.7 \\
Natural frequency $\omega_{n}$ & {$[\mathrm{rad} / \mathrm{s}]$} & 150 \\
\hline
\end{tabular}

To demonstrate the performance and robustness of the adaptive controller, uncertainties were introduced in the nonlinear model, which manifest themselves in the uncertain linear system as given in (1). The uncertainty is as follows: 
- Control effectiveness on all surfaces is reduced to $60 \%$ of the nominal value.

- Center of gravity is shifted 1.6 feet rearward, effectively representing an unknown center of pressure location.

- The rolling moment coefficient $C_{l}$ is reduced to $10 \%$ of the nominal value.

The simulation block diagram is shown in Figure 1.

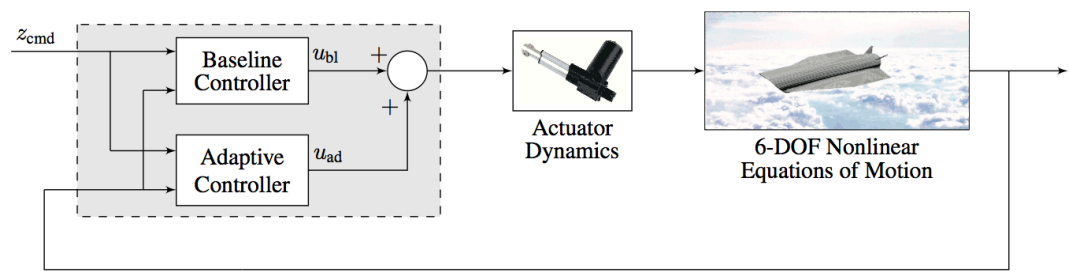

Figure 1. Simulation block diagram.

A 45 degree roll angle command was given, while commanding zero pitch rate. Figure 2 shows the response of the nominal system, with no uncertainty and only the baseline control law: $\Theta=0$. Figure 3 shows the response of the the system with the above uncertainty introduced and only the baseline controller: $\Theta=0$. Figure 4 shows the response of the system with the uncertainties when the adaptive augmentation is used.

The baseline control law applied to the nominal linear system provides good stability margins and closed-loop performance on the 6-DOF nonlinear evaluation model as shown in Figure 2. The system has a small rise time, minimal overshoot, and a small settling time. With the baseline control law only, introduction of the uncertainties causes greater initial overshoot, followed by significant high frequency oscillations, ultimately leading to instability and loss of the aircraft, as shown in Figure 3. The use of the adaptive component in the control law recovers the baseline control performance, as shown in Figure 4. When using the adaptive controller, both the control deflections and rates are well within acceptable limits.
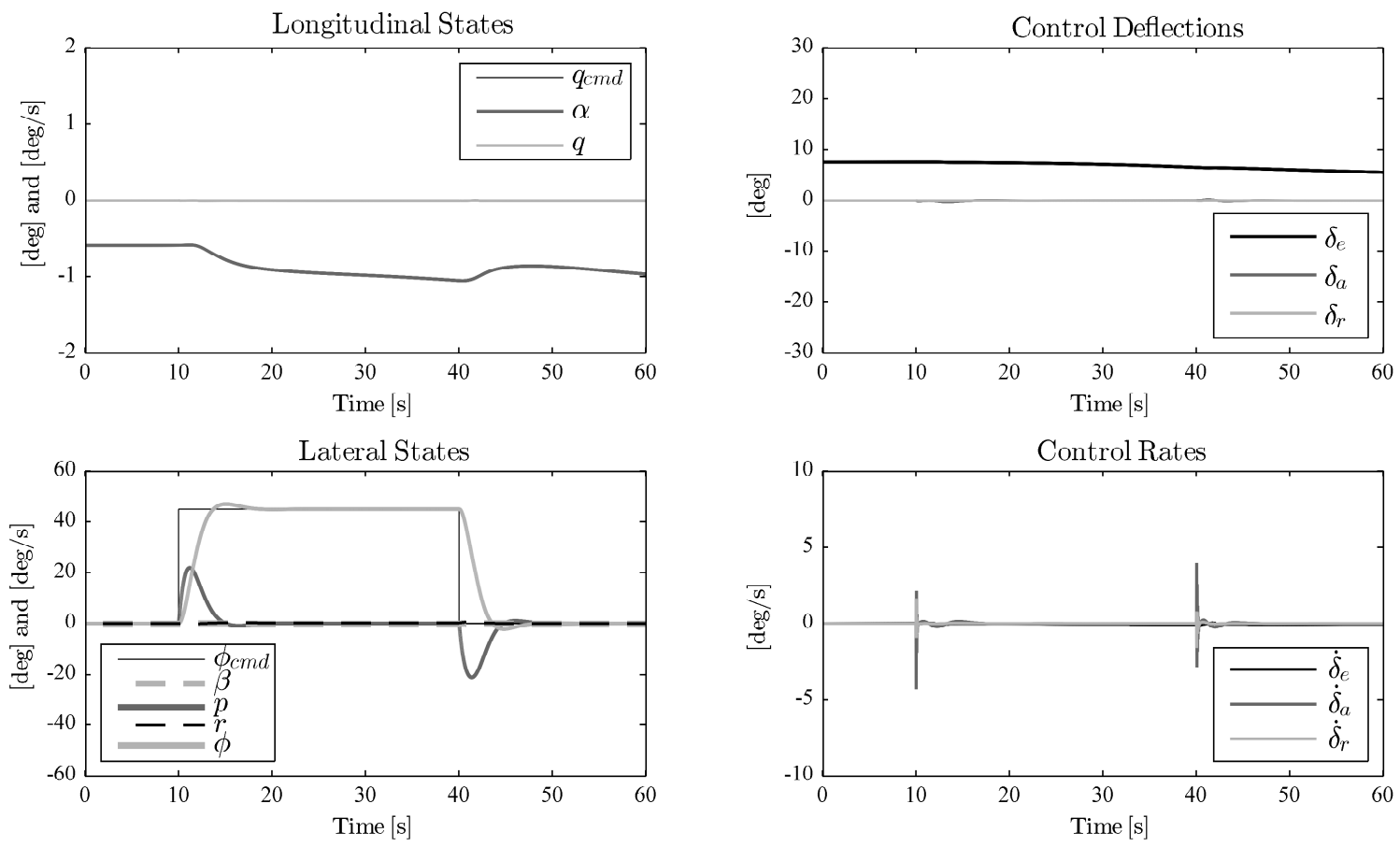

Figure 2. Time response of the nominal system: baseline controller with no uncertainty. 

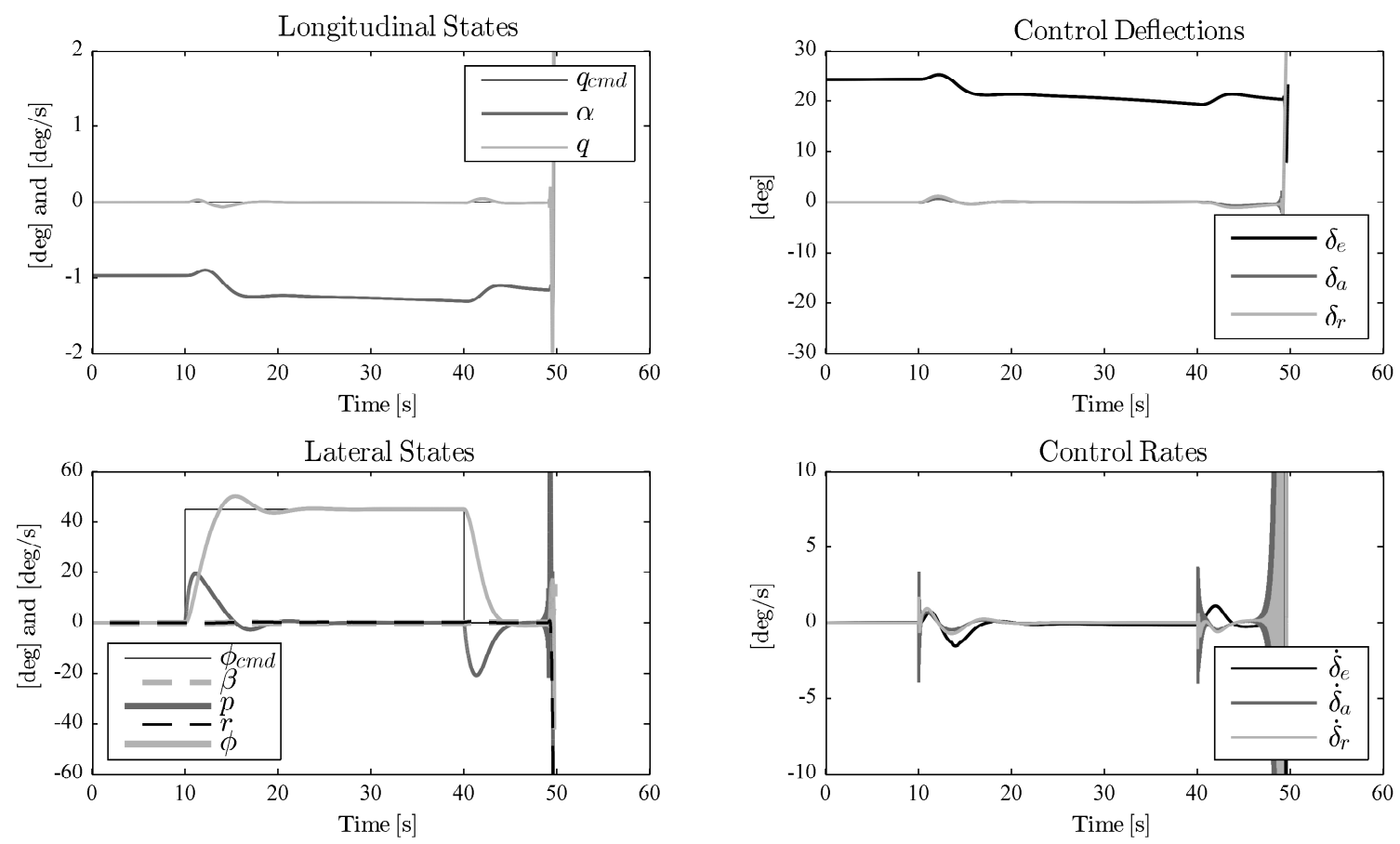

Figure 3. Time response of baseline control applied to uncertain plant.
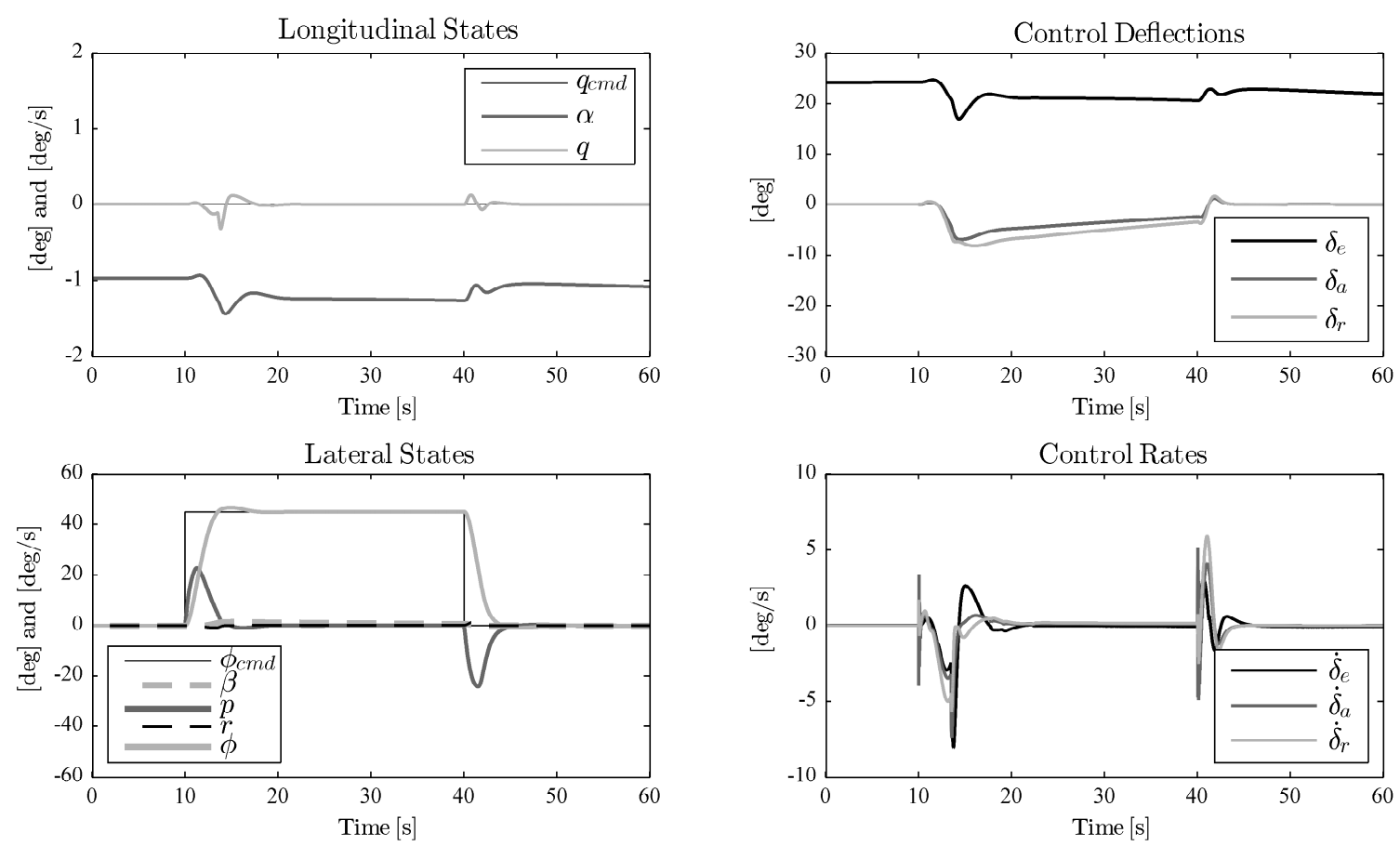

Figure 4. Time response of baseline controller with adaptive augmentation applied to uncertain plant. 


\section{Summary and Conclusions}

This paper has proposed a new alternative method for synthesizing a CRM based output feedback adaptive controller for a class of uncertain MIMO systems which do not have any unstable transmission zeros. The controller is composed of a baseline control gain augmented with an adaptive component to accommodate control effectiveness uncertainty and matched plant uncertainty, and makes use of the closed-loop reference model to improve the transient properties of the overall adaptive system. The adaptive controller requires the underlying error dynamics be made SPR through the synthesis of the postcompensator $S_{1}$ and CRM gain $L$, and the SPR relationship is enforced by reducing an underlying bilinear matrix inequality to a feasible linear matrix inequality through appropriate selection of a tuning matrix $X$. The procedure does not require the plant first be squared-up. It is computationally simple, and it requires only the calculation of some generalized inverses, the solution of the Lyapunov equation, and the solution of a reduced order state feedback problem. This procedure is summarized in nine straightforward steps. Furthermore, the degrees of freedom in the tuning matrix $X$ capture a large subset of all possible solutions which ensure the SPR property. Using these degrees of freedom, $X$ can be tuned to provide the desired stability margins for the baseline system, and a globally stable update law. The result is a baseline output feedback controller with good stability margins and adaptive augmentation capable of accommodating matched uncertainties.

This resulting robust baseline output feedback controller with adaptive augmentation is shown in simulation to provide good tracking performance when applied to a 6-DOF simulation of a hypersonic vehicle with significant uncertainty in control effectiveness, CG shift, and the rolling moment coefficient. The simulation results showed that for this uncertainty the robust baseline controller alone was unable to maintain stability, whereas the adaptive controller recovered nominal performance even with the presence of unmodeled actuator dynamics.

\section{Acknowledgment}

Approved for Public Release; Distribution Unlimited. Case Number 88ABW-2014-2551.

\section{References}

\footnotetext{
${ }^{1}$ Jang, J., Annaswamy, A. M., and Lavretsky, E., "Adaptive Flight Control in the Presence of Multiple Actuator Anomalies," American Control Conference, IEEE, New York, NY, July 2007, pp. 3300-3305.

${ }^{2}$ Gibson, T. E. and Annaswamy, A. M., "Adaptive Control of Hypersonic Vehicles in the Presence of Thrust and Actuator Uncertainties," AIAA Guidance, Navigation and Control Conference and Exhibit, American Institute of Aeronautics and Astronautics, Honolulu, HI, August 2008.

${ }^{3}$ Matsutani, M., Gibson, T. E., Jang, J., Crespo, L. G., and Annaswamy, A. M., "An Adaptive Control Technology for Safety of a GTM-like Aircraft," American Control Conference, IEEE, St. Louis, MO, June 2009, pp. 3238-3243.

${ }^{4}$ Crespo, L. G., Matsutani, M., Jang, J., Gibson, T. E., and Annaswamy, A. M., "Design and Verification of an Adaptive Controller for the Generic Transport Model," AIAA Guidance, Navigation, and Control Conference, AIAA, Chicago, IL, August 2009.

${ }^{5}$ Dydek, Z. T., Annaswamy, A. M., and Lavretsky, E., "Adaptive Control and the NASA X-15-3 Flight Revisited," IEEE Control Systems Magazine, Vol. 30, No. 3, June 2010, pp. 32-48.

${ }^{6}$ Wiese, D. P., Annaswamy, A. M., Muse, J. A., and Bolender, M. A., "Adaptive Control of a Generic Hypersonic Vehicle," AIAA Guidance, Navigation, and Control (GNC) Conference, AIAA, Boston, MA, August 2013.

${ }^{7}$ Lavretsky, E. and Wise, K. A., Robust and Adaptive Control, Springer, 2013.

${ }^{8}$ Singh, R. and Narendra, K. S., "Prior Information in the Design of Multivariable Adaptive Controllers," IEEE Transactions on Automatic Control, Vol. 29, No. 12, December 1984, pp. 1108-1111.

${ }^{9}$ Narendra, K. S. and Annaswamy, A. M., Stable Adaptive Systems, Dover Books on Electrical Engineering Series, Dover, 2005.

${ }^{10}$ Lavretsky, E., "Adaptive Output Feedback Design Using Asymptotic Properties of LQG / LTR Controllers," AIAA Guidance, Navigation, and Control Conference, AIAA, Toronto, Ontario, Canada, August 2010.

${ }^{11}$ Wise, K. A. and Lavretsky, E., "Flight Control Design Using Observer-based Loop Transfer Recovery," AIAA Guidance, Navigation, and Control (GNC) Conference, AIAA, Boston, MA, August 2013.

${ }^{12}$ Qu, Z., Lavretsky, E., and Annaswamy, A. M., "An Adaptive Controller for Very Flexible Aircraft," AIAA Guidance, Navigation, and Control (GNC) Conference, AIAA, Boston, MA, August 2013.

${ }^{13}$ Gibson, T. E., Annaswamy, A. M., and Lavretsky, E., "Improved Transient Response in Adaptive Control Using Projection Algorithms and Closed Loop Reference Models," AIAA Guidance, Navigation, and Control Conference, AIAA, Minneapolis, MN, August 2012.

${ }^{14}$ Gibson, T. E., Annaswamy, A. M., and Lavretsky, E., "Closed-loop reference models for output-feedback adaptive systems," European Control Conference (ECC), Zurich, Switzerland, July 2013, pp. 365-370.

${ }^{15}$ Gibson, T. E., Annaswamy, A. M., and Lavretsky, E., "On Adaptive Control With Closed-Loop Reference Models: Transients, Oscillations, and Peaking," IEEE Access, Vol. 1, September 2013, pp. 703-717.

${ }^{16}$ Gibson, T. E., Annaswamy, A. M., and Lavretsky, E., "Adaptive systems with closed-loop reference-models, part I: Transient performance," American Control Conference, IEEE, Washington DC, June 2013, pp. 3376-3383.
} 


\footnotetext{
${ }^{17}$ Misra, P., "A Computational Algorithm for Squaring-up Part I: Zero Input-Output Matrix," Proceedings of the 31st IEEE Conference on Decision and Control, IEEE, Tucson, AZ, December 1992, pp. 149-150.

${ }^{18}$ Qu, Z., Wiese, D. P., Annaswamy, A. M., and Lavretsky, E., "Squaring-up Method in the Presence of Transmission Zeros," The 19th World Congress of the International Federation of Automatic Control, IFAC, Cape Town, South Africa, August 2014, pp. 4164-4169.

${ }^{19}$ Rollins, E., Valasek, J., Muse, J. A., and Bolender, M. A., "Nonlinear Adaptive Dynamic Inversion Applied to a Generic Hypersonic Vehicle," AIAA Guidance, Navigation, and Control (GNC) Conference, AIAA, Boston, MA, August 2013.

${ }^{20}$ Huang, C., Ioannou, P. A., Maroulas, J., and Safonov, M. G., "Design of Strictly Positive Real Systems Using Constant Output Feedback," IEEE Transactions on Automatic Control, Vol. 44, No. 3, Mar 1999, pp. 569-573.

${ }^{21}$ Boyd, S. P., Ghaoui, L. E., Feron, E., and Balakrishnan, V., Linear Matrix Inequalities in System and Control Theory, Studies in Applied Mathematics, Society for Industrial and Applied Mathematics, 1994.

${ }^{22}$ Narendra, K. S. and Taylor, J. H., Frequency Domain Criteria for Absolute stability, Electrical science series, Elsevier Science, 1973.

${ }^{23}$ Kouvaritakis, B. and MacFarlane, A., "Geometric approach to analysis and synthesis of system zeros Part 1. Square systems," International Journal of Control, Vol. 23, No. 2, 1976, pp. 149-166.

${ }^{24}$ Barkana, I., "Comments on "Design of Strictly Positive Real Systems Using Constant Output Feedback"," IEEE Transactions on Automatic Control, Vol. 49, No. 11, Nov 2004, pp. 2091-2093.

${ }^{25}$ Owens, D., "Invariant zeros of multivariable systems: A geometric analysis," International Journal of Control, Vol. 26, No. 4, 1977, pp. 537-548.

${ }^{26}$ Kouvaritakis, B. and MacFarlane, A., "Geometric approach to analysis and synthesis of system zeros Part 2. Non-square systems," International Journal of Control, Vol. 23, No. 2, 1976, pp. 167-181.

${ }^{27}$ Antsaklis, P. J. and Michel, A. N., Linear systems, Springer, 2006.

${ }^{28}$ Chen, C., Introduction to Linear System Theory, Holt, Rinehart and Winston, 1970.

${ }^{29}$ Chen, C., Linear System Theory and Design, Oxford University Press, 1999.
}

\section{Appendix}

\section{Longitudinal Subsystem Numerical Data}

The nominal longitudinal plant matrices for a flight condition of Mach 6 at an altitude of 80,000 feet are:

$$
A_{p}=\left[\begin{array}{cc}
-0.2398 & 1.0000 \\
4.5689 & -0.1189
\end{array}\right] \quad B_{p}=\left[\begin{array}{c}
-0.0001 \\
-0.18561
\end{array}\right] \quad C_{p}=\left[\begin{array}{ll}
0 & 1
\end{array}\right] \quad C_{p z}=\left[\begin{array}{ll}
0 & 1
\end{array}\right]
$$

The following weighting matrices were used to compute $K_{x}$ as in (6) using the MATLAB command lqr

$$
\begin{aligned}
& Q_{\mathrm{lqr}}=\operatorname{diag}([0,0.3,170]) \\
& R_{\mathrm{lqr}}=0.0001
\end{aligned}
$$

\section{Lateral Subsystem Numerical Data}

The nominal lateral-directional plant matrices for a flight condition of Mach 6 at an altitude of 80,000 feet are:

$$
\begin{array}{rlrl}
A_{p} & =\left[\begin{array}{cccc}
-0.0699 & -0.0105 & -1.0000 & 0.0053 \\
-1331.0 & -2.0308 & -0.0076 & 0 \\
1.9465 & -0.0016 & -0.0533 & 0 \\
-0.0002 & 0.8536 & 0 & 0
\end{array}\right] & B_{p}=\left[\begin{array}{cc}
0 & 0.0002 \\
-8.1073 & 10.4560 \\
0.0320 & -0.2884 \\
0 & 0
\end{array}\right] \\
C_{p}=\left[\begin{array}{llll}
0 & 1 & 0 & 0 \\
0 & 0 & 1 & 0 \\
0 & 0 & 0 & 1
\end{array}\right] & C_{p z}=\left[\begin{array}{llll}
0 & 0 & 0 & 1
\end{array}\right]
\end{array}
$$

The following weighting matrices were used to compute $K_{x}$ as in (6) using the MATLAB command lqr

$$
\begin{aligned}
& Q_{\mathrm{lqr}}=\operatorname{diag}([0,0.2,40,0,0.2]) \\
& R_{\mathrm{lqr}}=\operatorname{diag}([0.005,0.005])
\end{aligned}
$$

